\title{
Article
}

\section{Solvent Extraction of Divalent Metal Ions Using 2-Pyridinealdoxime in Ionic Liquid}

\author{
*Kaoru Fujinaga, Kengo Kozaka, Yujiro Watanabe, and Yu Komatsu \\ Department of Environmental Chemistry, College of Environmental Engineering and Architecture, Kanazawa \\ Institute of Technology, \\ 3-1 Yatsukaho, Hakusan, Ishikawa 924-0838 Japan \\ Junji Noro \\ NISSAN ARC, LTD., 1 Natsushima-cho, Yokosuka, Kanagawa 237-0061 Japan
}

(Manuscript submitted March 30, 2007; accepted May 31, 2007)

\begin{abstract}
The extraction and separation of the divalent metals was studied by using ionic liquid, 1-butyl-3-methylimidazolium hexafluorophosphate $\left(\mathrm{BMIM}^{\left.-\mathrm{PF}_{6}\right)}\right.$ including the extractant of 2-pyridinealdoxime (2-PA). The divalent metals used here are $\mathrm{Cd}^{2+}$ and $\mathrm{Pb}^{2+}$ for the toxic and $\mathrm{Ni}^{2+}$ and $\mathrm{Zn}^{2+}$ for the valuable metals in industry. These metal ions in potassium nitrate of $0.10 \mathrm{~mol} \mathrm{dm}^{-3}$ concentration were examined to be extractable. It was observed that $\mathrm{Cd}^{2+}$ was extracted into ionic liquid phase better than $\mathrm{Pb}^{2+}$ and $\mathrm{Zn}^{2+}$ in the $\mathrm{pH}$ range of 5.15-9.83 and its extractability was reached to $>99 \%$ in the $\mathrm{pH}$ of 7.93-9.83. The extraction equilibrium of $\mathrm{Cd}^{2+}$ with 2-PA into BMIM-PF 6 was found to be $\mathrm{Cdcit}_{(\mathrm{w})}{ }^{-}+3 \mathrm{HL}_{(\mathrm{o})}$ $5 \mathrm{CdL}_{2} \mathrm{HL}_{(\mathrm{o})}+2 \mathrm{H}^{+}+\mathrm{cit}^{3-}$, whose mechanism is the same way as that into chloroform $\left(\mathrm{CHCl}_{3}\right)$. The mutual separation of $\mathrm{Cd}^{2+}, \mathrm{Pb}^{2+}, \mathrm{Ni}^{2+}$, and $\mathrm{Zn}^{2+}$ can be achieved by using the different $\mathrm{pH}$ values of the aqueous phase in 2-PA/ BMIM-PF ${ }_{6}$ extraction system. From these results, it can be stated that the 2-PA/ BMIM- $\mathrm{PF}_{6}$ extraction system is useful to extract and separate the divalent metal ions examined in this work.
\end{abstract}

Key words: solvent extraction, 2-Pyridinealdoxime, ionic liquid

\section{Introduction}

2-Pyridinealdoxime was found to be a selective extractant for $\mathrm{Cd}^{2+}$ and $\mathrm{Pb}^{2+}$ among the representative heavy metal ions by one of the present authors.[1,2] However, since 2-PA is fairly hydrophilic, of which its distribution constant between $\mathrm{CHCl}_{3}$ and water is $0.18,2$-PA can not be dissolved in ordinary hydrophobic solvents such as 1-octanol, cyclohexane, toluene, and n-hexane other than $\mathrm{CHCl}_{3}$, and these solvents can not be used for 2-PA extraction system. 2-PA is soluble in the solvents containing oxygen atom such as nitrobenzene, 4-methyl-2-pentanone, and ethyl acetate, but metal complexes with 2-PA are insoluble in these solvent and can not 
be extracted into these solvents. Recently, because of their environmentally harmfulness, halogenated organic solvents are avoided using. Consequently, 2-PA is not practical to use unless harmless solvent capable of dissolving 2-PA is found, although it has excellent selective extractability for $\mathrm{Cd}^{2+}$ and $\mathrm{Pb}^{2+}$.

Nowadays, new solvent, ionic liquids, attracts people's interests because of its nonvolatile and noncombustible character and is regarded to a solvent in harmony with environment. In the field of solvent extraction, ionic liquid is expected to be not only environment friendly solvent but also an extracting solvent

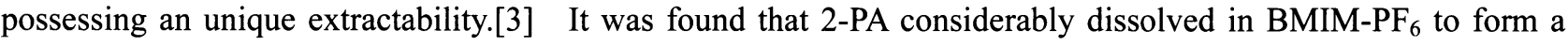
concentrated 2-PA solution, $0.10 M\left(M=\mathrm{mol} \mathrm{dm}^{-3}\right)$. In the present study, although nowadays there several kinds of ionic liquids are available, BMIM-PF 6 was mainly used in this study since it is used most widely and has a good solubility to 2-PA. $\mathrm{Ni}^{2+}, \mathrm{Cd}^{2+}, \mathrm{Pb}^{2+}$, and $\mathrm{Zn}^{2+}$ were arbitrarily selected as an analyte according to the environmental significance and were subjected to the extraction with 2-PA BMIM-PF 6 solution.

\section{Experimental}

\subsection{Reagents and apparatus}

A Varian model Vista-Pro ICP-AES spectrophotometer was used to measure the metal ion concentration and a Horiba F-21 pH meter was used to measure pH. 2-PA was obtained from Tokyo Kasei Kogyo Co. Ltd. and was purified by recrystallization from chloroform. The other reagents were all of analytical grade.

\subsection{Extraction procedure}

A $2.0 \mathrm{ml}$ portion of $0.20 \mathrm{mM}$ metal ion solution, which was masked and buffered with $0.010 \mathrm{M}$ tripotassium citrate $\left(\mathrm{K}_{3} \mathrm{cit}\right)$ and was maintained ionic strength to be $0.10 \mathrm{M}$ with potassium nitrate, was equilibrated with an

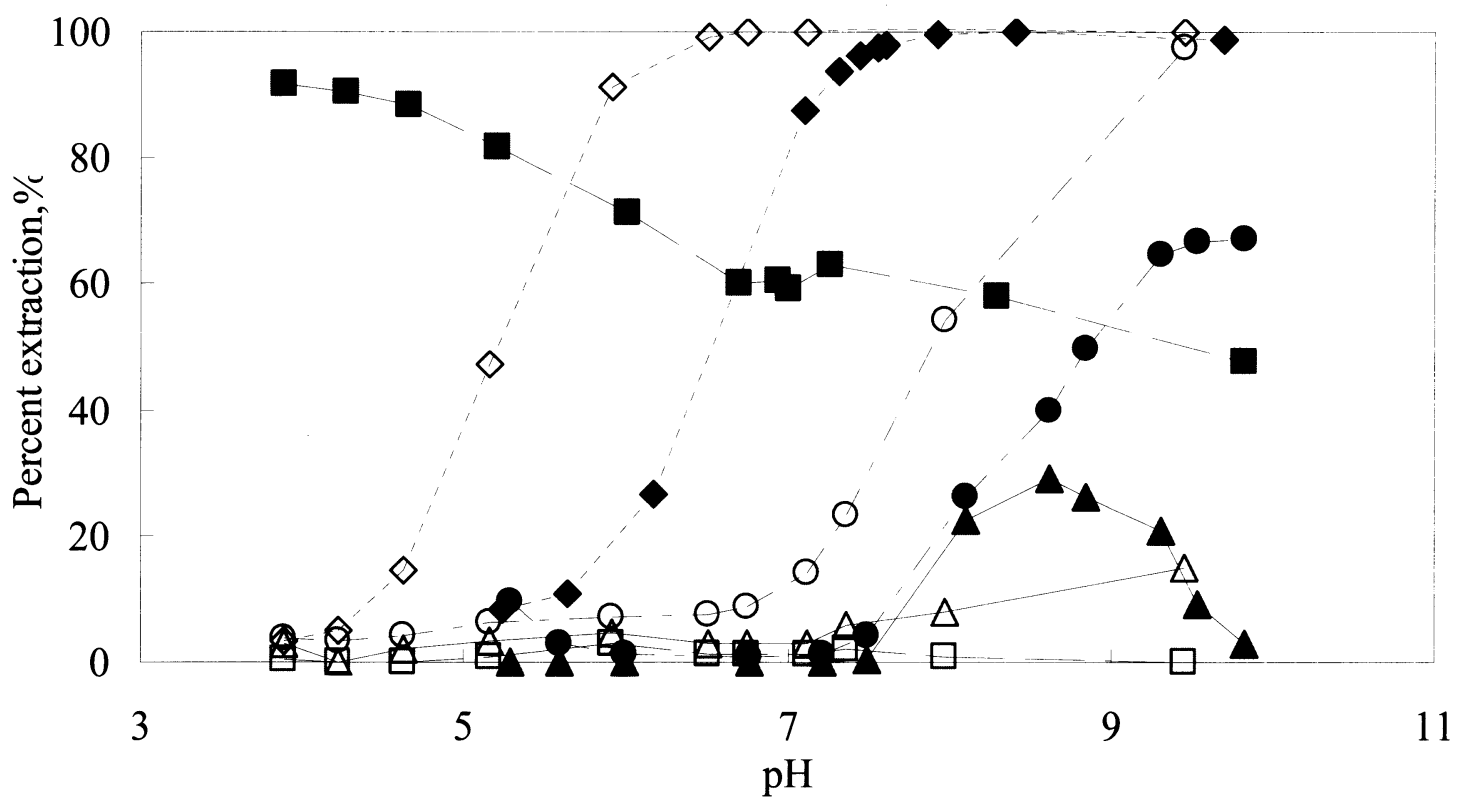

Fig.1 Distribution ratios of some divalent metals as a function of $\mathrm{pH}$.

Organic phase: $[2-\mathrm{PA}]_{\mathrm{BMIM}-\mathrm{PF} 6}=0.10 \mathrm{M}$. Aqueous phase: $\left[\mathrm{M}^{2+}\right]=0.20 \mathrm{mM},\left[\mathrm{KNO}_{3}\right]=0.10 \mathrm{M},\left[\mathrm{K}_{3} \mathrm{cit}\right]=0.010 \mathrm{M}$.

$\mathrm{Pb}^{2+}, \ldots .-\mathrm{Cd}^{2+}, \ldots \ldots \ldots . . . . \mathrm{Ni}^{2+},--\mathbf{a}_{--} ;$and $\mathrm{Zn}^{2+}$,

—_ for BMIM-PF 6 extraction system:

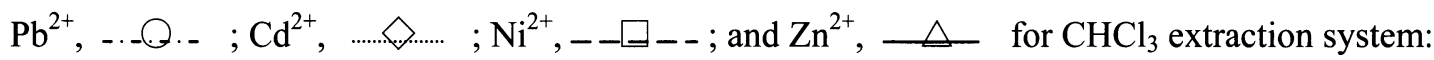


equal volume of $0.10 \mathrm{M}$ 2-PA BMIM-PF ${ }_{6}$ solution in a $10 \mathrm{ml}$ extraction vial by shaking vigorously for $1 \mathrm{~h}$, followed by separating phases by centrifugation. After determining the equilibrated $\mathrm{pH}$ of aqueous phase, $1.0 \mathrm{ml}$ of the BMIM-PF 6 phase was subjected to the back-extraction with $1.0 \mathrm{ml}$ of $1.0 \mathrm{M} \mathrm{HCl}$ solution for $1 \mathrm{~h}$. Then, the metal ion concentrations in both aqueous phases were determined by ICP-AES, and the extraction ratio was obtained from the determined metal ion concentrations in both aqueous phases. To evaluate the extractability of BMIM-PF 6 , metal extraction was carried out separately by using $\mathrm{CHCl}_{3}$ as an extracting solvent under the same extraction conditions.

\section{Results and Discussion}

\subsection{Effect of pH}

In the previously reported 2-PA $\mathrm{CHCl}_{3}$ extraction system, only $\mathrm{Cd}^{2+}$ and $\mathrm{Pb}^{2+}$ were extracted into organic phase, while others were not. As for $\mathrm{Cd}^{2+}, \mathrm{Pb}^{2+}$, and $\mathrm{Zn}^{2+}$, roughly speaking, the extraction curves obtained by BMIM-PF ${ }_{6}$ extraction system looked similar to those obtained by the 2-PA $\mathrm{CHCl}_{3}$ extraction system, but there are several differences in detail. First, in the present extraction system, $\mathrm{pH}_{50 \%}$ values were 6.45 for $\mathrm{Cd}^{2+}$ and 8.86 for $\mathrm{Pb}^{2+}$, respectively, and were worse compared to those in the 2-PA $\mathrm{CHCl}_{3}$ extraction system, 5.15 for $\mathrm{Cd}^{2+}$ and 7.89 for $\mathrm{Pb}^{2+}$. Second, the maximum of extraction curve for $\mathrm{Pb}^{2+}$ did not reach to $100 \%$ in the present system as seen in Fig.1, while that attained to more than $99 \%$ in the 2-PA $\mathrm{CHCl}_{3}$ extraction system. The worse extractability of BMIM- $\mathrm{PF}_{6}$ for these metal 2-PA complexes compared to $\mathrm{CHCl}_{3}$ may be attributed to its poor solubility, if these metals were extracted in the form of 2-PA chelate complexes, as described in section 3.2. BMIM-PF 6 does not dissolve in $\mathrm{CHCl}_{3}$ or in water mutually and form three layers when mixed them. Therefore, chemical properties such as polarity are thought to be fairly different each other between $\mathrm{CHCl}_{3}$ and $\mathrm{BMIM}-\mathrm{PF}_{6}$.

As for $\mathrm{Ni}^{2+}$, there is a striking contrast in the extraction behavior with between $\mathrm{CHCl}_{3}$ and $\mathrm{BMIM}-\mathrm{PF}_{6}$. That is, the percent extraction of $\mathrm{Ni}^{2+}$ was less than $3 \%$ in the entire $\mathrm{pH}$ region examined in the 2-PA $\mathrm{CHCl}_{3}$ extraction system, but that was greater than $90 \%$ in the $\mathrm{pH}$

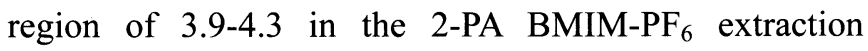
system.

\subsection{Extraction equilibrium}

It is known that components in ionic liquid react

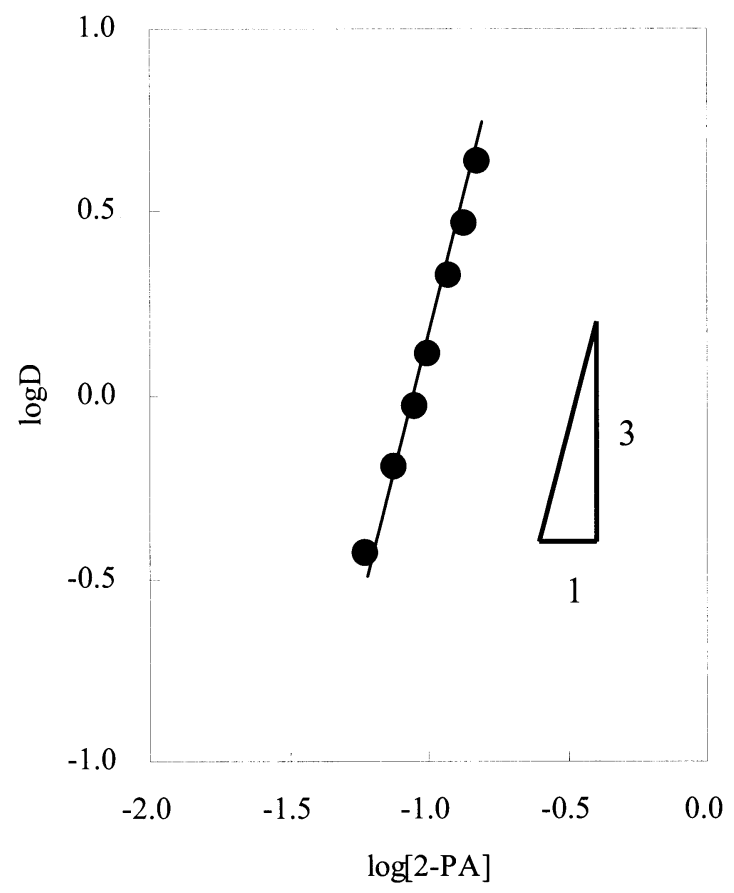
with metal chelate complex to form an ion association complex in the some metal extraction systems.[4] Therefore, the extraction of these metals with $\mathrm{CHCl}_{3}$ solution containing 1-butyl-3-methyl imidazolium chloride and 2-PA and the extraction of these metals

Fig.2 Distribution ratio of $\mathrm{Cd}^{2+}$ as a function of 2-PA concentration.

$\mathrm{pH}$ was maintained at $6.64 \pm 0.05$. 
from aqueous phase containing hexafluorophosphoric acid into 2-PA $\mathrm{CHCl}_{3}$ solution were examined. The both experimental results showed that the components of $\mathrm{BMIM}-\mathrm{PF}_{6}$ did not affect 2-PA $\mathrm{CHCl}_{3}$ extraction at all. Therefore, it was considered that $\mathrm{BMIM}-\mathrm{PF}_{6}$ worked simply as a diluent and metals were extracted in the form of chelate complex without forming ion association complex.

In the 2-PA $\mathrm{CHCl}_{3}$ extraction system, it was ascertained by the slope analysis that $\mathrm{Cd}^{2+}$ was extracted in accordance with the following equilibrium,

$\mathrm{Cdcit}_{(\mathrm{w})}{ }^{-}+3 \mathrm{HL}_{(\mathrm{o})} \leftrightarrows \mathrm{CdL}_{2} \mathrm{HL}_{(\mathrm{o})}+2 \mathrm{H}^{+}+\mathrm{cit}^{3-}$

where the subscript (o) denotes the organic phase and (w) means the aqueous phase and Cdcit and $\mathrm{cit}^{3-}$ are the cadmium citrate complex and citrate anion, respectively. If same things hold in the present 2-PA BMIM-PF 6 extraction system, the plots of $\log \mathrm{D} v$ s. $\mathrm{pH}$ and $\log \mathrm{D} v$ s. $\log [2-\mathrm{PA}]$ for $\mathrm{Cd}^{2+}$ should produce the line with slopes of 2 and 3, respectively. The plots of $\log \mathrm{D} v$. $\mathrm{pH}$ for $\mathrm{Cd}^{2+}$, which was obtained from the data shown in Fig.1, exhibited slope of $1.9\left(r^{2}=0.981\right)$, and the plots of $\log \mathrm{D} v$. $\log [2-\mathrm{PA}]$ showed slope of $2.9\left(\mathrm{r}^{2}=0.997\right)$, as can be seen in Fig.2. Since the slopes of the $\log \mathrm{D} v s$. pH and $\log \mathrm{D} v s$. $\log [2-\mathrm{PA}]$ plots are close to 2 and 3, it can be concluded that 2-PA BMIM-PF 6 solution extracted $\mathrm{Cd}^{2+}$ in the same way to the 2-PA $\mathrm{CHCl}_{3}$ extraction system. $\mathrm{As}$ for $\mathrm{Pb}^{2+}$ and $\mathrm{Zn}^{2+}$, neither metals were extracted sufficiently, so that further examination was not carried out in this study. While, the slope of the $\log \mathrm{D}$ s. $\mathrm{pH}$ plots of $\mathrm{Ni}^{2+}$ was -0.2 , and did not give the integral number. Although it was suspected that components in ionic liquid react with Ni-complex with 2-PA to form an adduct, details have not been proved yet.

\subsection{Separation between $\mathrm{Ni}^{2+}, \mathrm{Cd}^{2+}, \mathrm{Pb}^{2+}$, and $\mathrm{Zn}^{2+}$}

In the present 2-PA BMIM-PF 6 extraction system, extraction behavior of $\mathrm{Ni}^{2+}$ was quite different from those of $\mathrm{Cd}^{2+}, \mathrm{Pb}^{2+}$, and $\mathrm{Zn}^{2+}$, so that $\mathrm{Ni}^{2+}$ can be easily separated from these metal ions by carrying out its extraction in the acidic $\mathrm{pH}$ region. At $\mathrm{pH} 7.2$, percent extractions of $\mathrm{Pb}^{2+}$ and $\mathrm{Zn}^{2+}$ were both $0 \%$, while that of $\mathrm{Cd}^{2+}$ was $92 \%$, therefore, $\mathrm{Cd}^{2+}$ can also be fully separated from $\mathrm{Pb}^{2+}$ and $\mathrm{Zn}^{2+}$ by extracting $\mathrm{Cd}^{2+}$ at $\mathrm{pH}$ 7.2. The separation of $\mathrm{Pb}^{2+}$ and $\mathrm{Zn}^{2+}$ is also possible by extracting them at $\mathrm{pH} 9.8$, since the extractabilities of $\mathrm{Pb}^{2+}$ and $\mathrm{Zn}^{2+}$ at $\mathrm{pH} 9.8$ were $67 \%$ and $3 \%$, respectively.

In conclusion, it can be said that the separation between $\mathrm{Ni}^{2+}, \mathrm{Cd}^{2+}, \mathrm{Pb}^{2+}$, and $\mathrm{Zn}^{2+}$ can be achieved by the present 2-PA BMIM-PF 6 extraction system.

\section{References}

1. K. Fujinaga, Y. Sakaguchi, T. Tsuruhara, Y. Seike, and M. Okumura, Solvent Extr. Res. Dev., Jpn., 8, 144-158 (2001).

2. K. Fujinaga, H. Nagura, R. Yamasaki, H. Kokusen, Y. Komatsu, Y. Seike, and M. Okumura, Solvent Extr. Res. Dev., Jpn., 13, 175-184 (2006).

3. J. L. Anderson, D. W. Armstrong, and Guor-Tzo Wei, Anal. Chem., 78, 2892-2902 (2006).

4. N. Hirayama, Bunseki, 382, 519-523 (2006). 